\title{
Non-local dynamics governing the self-induced motion of a planar vortex filament
}

\author{
Robert A. Van Gorder* \\ Oxford Centre for Industrial and Applied Mathematics \\ Mathematical Institute, University of Oxford \\ Andrew Wiles Building, Radcliffe Observatory Quarter, Woodstock Road \\ Oxford OX2 6GG United Kingdom \\ ${ }^{*}$ Corresponding author. Email: Robert.VanGorder@maths.ox.ac.uk
}

May 24, 2015

\begin{abstract}
While the Hasimoto planar vortex filament is one of few exact solutions to the local induction approximation (LIA) approximating the self-induced motion of a vortex filament, it is natural to wonder whether such a vortex filament solution would exist for the non-local Biot-Savart dynamics exactly governing the filament motion, and if so, whether the non-local effects would drastically modify the solution properties. Both helical vortex filaments and vortex rings are known to exist under both the LIA and non-local Biot-Savart dynamics, however the planar filament is a bit more complicated. In the present paper, we demonstrate that a planar vortex filament solution does exist for the non-local Biot-Savart formulation, provided that a specific non-linear integral equation (governing the spatial structure of such a filament) has a non-trivial solution. By using the Poincaré - Lindstedt method, we are able to obtain an accurate analytical approximation to the solution of this integral equation under physically reasonable assumptions. To obtain these solutions, we approximate local effects near the singularity of the integral equation using the LIA, and non-local effects using the Biot-Savart formulation. Mathematically, the results constitute an analytical solution to an interesting nonlinear singular integro-differential equation in space and time variables. Physically, these results show that planar vortex filaments exist and maintain their form under the non-local Biot-Savart formulation, as one would hope. Due to the regularization approach utilized, we are able to compare the structure of the planar filaments obtained under both LIA and Biot-Savart formulations in a rather straightforward manner, in order to determine the role of the non-locality on the structure of the planar filament.
\end{abstract}

Keywords: Vortex dynamics; vortex filament; non-local equation; Biot-Savart dynamics; planar filaments

\section{Introduction}

The self-induced motion of a thin vortex filament is commonly studied by use of the the Biot-Savart law. However, since the Biot-Savart law involves an integral term with logarithmic singularity, it is very common to apply the local induction approximation (LIA) in order to approximate the self-induced motion of such a vortex filament. This approximation takes the integral equation 
corresponding to the Biot-Savart law and reduces it into a vector partial differential equation, the solution of which gives the time evolution of the position of the vortex filament in space. Corresponding to this approximation, the classical Da Rios equations for the motion of a vortex filament in a standard fluid $[1,2]$ have been studied for a wide variety of vortex configurations. 1-solitons on a filament[3], torus knots [4] (with Biot-Savart dynamics of torus knots considered in [5]), helical filaments [6], planar filaments [8], and self-similar structures [9] are some of the solutions found, illustrating the different physical scenarios possible. One reason that the LIA is of interest is that it is a limiting case (the zero-temperature limit) for the motion of a vortex filament in a superfluid [10, 11].

One important and rather fundamental solution to the LIA, the planar vortex filament, was first found by Da Rios [2, 7] and also studied later by Hasimoto [8]. The solution takes the form of a plane curve, with the motion being rotational. One fundamental property of this filament solution is its relation to elastica, as discussed by Hasimoto. The planar solution was cast in a wider setting by Kida [4], who was able to compare it with other types of solutions. Mathematically, the planar filament is a type of stationary solution for the partial differential equation resulting from the LIA, in that the filament moves (again, through rotation) but does not undergo a change in structure. It is possible to obtain the planar filament directly through Cartesian coordinates, see [12]. The Poincaré - Lindstedt method was used to obtain the spatial structure of a planar filament in the Cartesian frame by Van Gorder [13]. In this approach, deviations from a centrally aligned reference axis were taken to be the small parameter, and an approximate closed form of the solutions was given without the need for the inversion of elliptic integrals (which would be required for an exact representation). Kida performed a type of numerical stability analysis on this type of filament [14], while Van Gorder [15] was able to obtain an analytical stability result for the orbital stability (spectral stability) of such filaments. Fukumoto [16] studied the influence of background flows on planar filaments. We should also note that, in addition to the standard curvature-torsion frame (used by Hasimoto) or the Cartesian frame, there is an alternate formulation, given by Umeki [17], which formulates the LIA in terms of the unknown tangent vector as a function of arclength and time. A planar solution in this framework was given by Van Gorder [18].

While the planar filament is well-studied under the dynamics resulting from the LIA, it is natural to wonder whether such a vortex filament solution would exist for the full non-local Biot-Savart law, and if so, whether the non-local effects would drastically modify the solution properties. This issue has not been addressed before in the literature, and hence shall be the focus of this paper. We first show that the question of the existence of a planar vortex filament solution for the non-local Biot-Savart formulation governing the self-induced motion of a vortex filament can be recast into a question of whether a solution exists for a kind of singular and nonlinear integral equation involving an unknown function which shall give the spatial profile of the planar filament. A general planar filament is of the form $\mathbf{r}=(x, \cos (\omega t) \psi(x), \sin (\omega t) \psi(x))$, and we demonstrate that the unknown function $\psi(x)$ (which gives the shape of the planar filament) is governed by

$$
\omega \psi(x)=\frac{\Gamma}{4 \pi} \int_{\mathbb{R}} \frac{(s-x) \psi^{\prime}(s)-(\psi(s)-\psi(x))}{\left((s-x)^{2}+(\psi(s)-\psi(x))^{2}\right)^{3 / 2}} d s .
$$

Observe that determining the planar filament in the Biot-Savart formulation is more challenging than for other known solutions, such as the helical filament. For instance, it can be shown that the helical filament $\mathbf{r}(x, t)=\left(x+\beta t, A \cos \left(k x-\omega t+x_{0}\right), A \sin \left(k x-\omega t+x_{0}\right)\right)$ can be solved for exactly under the Biot-Savart formulation, in the sense that one can recover the motion parameters 
$\beta$ and $\omega$ in terms of the other model parameters. The need to solve the integral equation governing the planar filament makes the planar solution much harder to study than other solutions, under the Biot-Savart formulation. Also, the shape of a planar filament under LIA is given by an ordinary differential equation, which again is much simpler to work with than an integral equation. Therefore, the planar filament will be more challenging to study under Biot-Savart dynamics than under the LIA.

Due to the nonlinearity and singularity inherent in the integral equation, an exact solution is not possible to obtain. We therefore resort to an analytical method, valid for filaments with sufficiently small deviations from the reference axis, to describe the properties of the solution. We find that the LIA and Biot-Savart formulations both admit solutions with very similar spatial structures, which makes sense in light of the fact that the spatial structure is unchanging in time. We see that the non-locality inherent in the Biot-Savart formulation primarily influences the rotational motion of the filament. Therefore, we show that planar vortex filament solutions previously discussed in the context of the LIA can actually exist for the more complicated Biot-Savart formulation. Structurally the LIA and Biot-Savart planar filaments are quite similar.

\section{Biot-Savart formulation for a parameterized filament curve}

Before moving on to the planar filament, let us consider a general line filament under the BiotSavart formulation. Let the curve $\mathbf{r}$ represent the vortex filament in Cartesian coordinates. The self-induced motion of a thin vortex filament is given by the Biot-Savart law $[19,20,7]$

$$
\mathbf{r}_{t}=\frac{\Gamma}{4 \pi} \int_{\ell} \frac{(\mathbf{s}-\mathbf{r}) \times d \mathbf{s}}{|\mathbf{s}-\mathbf{r}|^{3}}
$$

Here, $\mathbf{r}$ denotes a space point in $\mathbb{R}^{3}$ lying on the filament, the subscript $t$ denotes the time derivative, $\Gamma$ denotes the circulation, and $\ell$ is the path traced out along the filament. This representation is non-local, meaning that distant parts of the filament can effect the behavior of the filament at a specific point. Since this equation is non-local, it is difficult to solve for most vortex configurations. Often, the LIA is used to replace the integral in (1) with a far simpler relation, viz.,

$$
\mathbf{r}_{t}=\frac{\Gamma}{4 \pi} \ln (1 / \epsilon) \kappa \mathbf{t} \times \mathbf{n},
$$

where $\mathbf{t}$ is the tangent vector and $\mathbf{n}$ denotes the normal vector to $\mathbf{r}, \kappa$ is the local curvature, and $\epsilon$ denotes a small length parameter arising from the fact that we approximate the Biot-Savart dynamics in a neighborhood of the singularity. The right hand side of (2) is the binormal vector (up to scaling).

In the present paper, we shall be interested in a Cartesian representation of a vortex filament, so let us write $\mathbf{r}=(X, Y, Z)$. Equation (1) gives the vector relation

$$
\left(X_{t}, Y_{t}, Z_{t}\right)=\frac{\Gamma}{4 \pi} \int_{\ell} \frac{\left(S_{1}-X, S_{2}-Y, S_{3}-Z\right) \times\left(d S_{1}, d S_{2}, d S_{3}\right)}{\left[\left(S_{1}-X\right)^{2}+\left(S_{2}-Y\right)^{2}+\left(S_{3}-Z\right)^{2}\right]^{3 / 2}} .
$$

This gives the time evolution for each component of $\mathbf{r}$. If we desire a solution curve $\mathbf{r}$ to the BiotSavart law (1), we must be able to solve (3) for the position components. Depending on the type of solution sought, solving this system may vary from difficult to impossible. 
Note that (3) is singular when $\left(S_{1}, S_{2}, S_{3}\right)=(X, Y, Z)$, which further complicates the solution of such a model. To overcome this, we shall apply the LIA to the region near $(X, Y, Z)$, while we shall maintain the full Biot-Savart law outside of such a region. In other words, the time evolution of the solution curve $\mathbf{r}=(X, Y, Z)$ shall consist of one component giving a local effect very near each point on the curve (the LIA term) and one component giving the non-local effect (sufficiently removed from the point).

If we assume that the vortex filament is essentially aligned along one axis and there is no motion along the reference axis (which is always true in the case of a planar filament, since the motion of such a filament is purely rotational) then we may parameterize the filament by $X=x$, $Y=Y(x, t), Z=Z(x, t)$. Compare this to the helical filament, for which one needs to consider $X=X(x, t)=x+\beta t$ to account for translation along the reference axis. Taking $s$ to denote a variable of integration, (3) then reduces to

$$
\left(0, Y_{t}, Z_{t}\right)=\frac{\Gamma}{4 \pi} \int_{\ell} \frac{(s-x, Y(s, t)-Y(x, t), Z(s, t)-Z(x, t)) \times\left(d s, Y_{x}(s, t) d s, Z_{x}(s, t) d s\right)}{\left[(s-x)^{2}+(Y(s, t)-Y(x, t))^{2}+(Z(s, t)-Z(x, t))^{2}\right]^{3 / 2}} .
$$

\section{An integral equation for the non-local planar vortex filament}

Recall that under the LIA the planar vortex filament takes the form

$$
\mathbf{r}(x, t)=(x, \cos (\omega t) \psi(x), \sin (\omega t) \psi(x)),
$$

where $\psi$ is a real-valued, twice differentiable function of $x$ alone. The constant $\omega$ is a parameter to be determined, which has the interpretation of giving the rotational velocity of the filament. The solution (5) is interesting, in that it describes a rotating filament which is aligned along a plane intersecting the $x$-axis. Such a filament, first described by Hasimoto under the LIA, is also interesting due to its relation to elastica. What we shall do in this section is derive conditions for a non-local analogue to the Hasimoto planar filament to exist.

Assuming a solution of the form (5), the time evolution due to the Biot-Savart formulation (1) is

$$
\mathbf{r}_{t}(x, t)=\frac{\Gamma}{4 \pi} \int_{\mathbb{R}} \frac{(\mathbf{r}(x, t)-\mathbf{r}(s, t)) \times\left(d s, \cos (\omega t) \psi^{\prime}(s) d s, \sin (\omega t) \psi^{\prime}(s) d s\right)}{|\mathbf{r}(s, t)-\mathbf{r}(x, t)|^{3}} .
$$

We calculate

$$
\begin{gathered}
|\mathbf{r}(s, t)-\mathbf{r}(x, t)|^{3}=\left((s-x)^{2}+\cos ^{2}(\omega t)(\psi(s)-\psi(x))^{2}+\sin ^{2}(\omega t)(\psi(s)-\psi(x))^{2}\right)^{3 / 2} \\
=\left((s-x)^{2}+(\psi(s)-\psi(x))^{2}\right)^{3 / 2}, \\
(\mathbf{r}(x, t)-\mathbf{r}(s, t)) \times\left(d s, \cos (\omega t) \psi^{\prime}(s) d s, \sin (\omega t) \psi^{\prime}(s) d s\right) \\
=\left(0, \sin (\omega t)\left\{(\psi(s)-\psi(x))-(s-x) \psi^{\prime}(s)\right\} d s,-\cos (\omega t)\left\{(\psi(s)-\psi(x))-(s-x) \psi^{\prime}(s)\right\} d s\right),
\end{gathered}
$$

and

$$
\mathbf{r}_{t}(x, t)=(0,-\omega \sin (\omega t) \psi(x), \omega \cos (\omega t) \psi(x)) .
$$

Placing these expressions into (6), we obtain the equations $X: 0=0$,

$$
Y: \quad-\omega \sin (\omega t) \psi(x)=\sin (\omega t) \frac{\Gamma}{4 \pi} \int_{\mathbb{R}} \frac{(\psi(s)-\psi(x))-(s-x) \psi^{\prime}(s)}{\left((s-x)^{2}+(\psi(s)-\psi(x))^{2}\right)^{3 / 2}} d s,
$$


and

$$
Z: \quad \omega \cos (\omega t) \psi(x)=-\cos (\omega t) \frac{\Gamma}{4 \pi} \int_{\mathbb{R}} \frac{(\psi(s)-\psi(x))-(s-x) \psi^{\prime}(s)}{\left((s-x)^{2}+(\psi(s)-\psi(x))^{2}\right)^{3 / 2}} d s,
$$

for the $X, Y$, and $Z$ components, respectively. Both of these equations are equivalent, so we obtain the single equation

$$
\omega \psi(x)=\frac{\Gamma}{4 \pi} \int_{\mathbb{R}} \frac{(s-x) \psi^{\prime}(s)-(\psi(s)-\psi(x))}{\left((s-x)^{2}+(\psi(s)-\psi(x))^{2}\right)^{3 / 2}} d s .
$$

For simplicity of calculations, we shall define the composite parameter

$$
\lambda=-\frac{\Gamma}{4 \pi \omega},
$$

so that (12) becomes

$$
\psi(x)+\lambda \int_{\mathbb{R}} \frac{(s-x) \psi^{\prime}(s)-(\psi(s)-\psi(x))}{\left((s-x)^{2}+(\psi(s)-\psi(x))^{2}\right)^{3 / 2}} d s=0 .
$$

From previous work $[13,15]$, we know that we should have the spectral parameter $\omega<0$, so $\lambda>0$. Equation (14) constitutes a non-linear and singular integral equation for the unknown function $\psi(x)$. Note that this equation does not depend on time (which makes sense, as the planar filament is a type of stationary solution). While superficially the integral appears to be singular with a singularity of degree three, note that the integrand actually has a first degree singularity. Indeed, expanding the integrand about $s=x$, we find

$$
\begin{aligned}
\frac{(s-x) \psi^{\prime}(s)-(\psi(s)-\psi(x))}{\left((s-x)^{2}+(\psi(s)-\psi(x))^{2}\right)^{3 / 2}} & =\frac{1}{2} \frac{\psi^{\prime \prime}(x)}{\left(1+\psi^{\prime 2}(x)\right)^{3 / 2}} \frac{1}{|s-x|} \\
& +\left(\frac{1}{3} \frac{\psi^{\prime \prime \prime}(x)}{\left(1+\psi^{\prime 2}(x)\right)^{3 / 2}}-\frac{3}{4} \frac{\psi^{\prime}(x) \psi^{\prime \prime 2}(x)}{\left(1+\psi^{\prime 2}(x)\right)^{5 / 2}}\right) \operatorname{sgn}(s-x)+O(|s-x|) .
\end{aligned}
$$

Therefore, the integral (14) itself should have a singularity of strength $\ln (|s-x|)$ for $|s-x|<<1$. Since (14) is non-linear and singular, this is little hope in obtaining an exact solution, and even numerical methods will be difficult to apply due to the form of the nonlinear dependence of the integrand on $\psi$. In the next section, we apply the method of multiple scales to the solution of the integral equation (14). In order to avoid the singularity at $s=x$, we apply the LIA for $|s-x|<\epsilon$, for some small $\epsilon$. Then, for $|s-x|>\epsilon$, we keep the Biot-Savart integral term. This results in an extension of the LIA results which accounts for non-local effects. This type of regularization approach was recently applied to the non-local helical vortex filament (and will appear in a forthcoming work).

Clearly, (14) is identically satisfied by the zero solution $\psi(x)=0$, corresponding to the line filament $\mathbf{r}=(x, 0,0)$. In other words, we recover the line filament when the planar deflections tend to zero, as expected (and as would be required of an appropriate formulation). Alternately, if the spectral parameter $\omega=0$, then $\psi(x)=\alpha x$ is an exact solution of (14) (for any non-zero constant $\alpha)$. This results in a slanted line filament $\mathbf{r}(x, t)=(x, \alpha x, 0)$. The interesting case is where the maximal deflection from the $x$ axis is of bounded amplitude $A$, where $A=\max \psi(x)>0$. Such solutions, as we shall later see, correspond to space-periodic functions $\psi(x)$, and these are the direct analogue to the planar solutions of the LIA which may be given in terms of elliptic integrals. We shall study such space-periodic solutions of (14) in the following section. 


\section{Approximating the non-local planar vortex filament}

In this section, we obtain an approximate analytical solution to the integral equation (14). Once a solution $\psi$ is obtained, we will have recovered the planar vortex filament (5). We shall split the Biot-Savart integral into two parts, corresponding to both $|s-x|<\epsilon$ and $|s-x|>\epsilon$, where $\epsilon$ is a small length parameter. For such small $\epsilon$, the contribution on $|s-x|<\epsilon$ is adequately approximated by the LIA. The method was recently employed to study the Biot-Savart helical filament and to compare it directly with the LIA helical filament [21]. For the helical filaments, note that one can describe the filaments in terms of constants for the rotational and translational velocity, with each quantity being given in terms of integrals which depend on the amplitude and wave number. In contrast, the planar filament is determined by an unknown function which is governed by an integral equation. Hence, the planar filament is a bit more challenging to describe mathematically, since this unknown function is harder to solve for than the unknown parameters needed to describe the helical filament.

For a planar filament of the form (5), the LIA gives

$$
-\ln (\epsilon) \frac{\psi^{\prime \prime}(x)}{\left(1+\psi^{\prime 2}(x)\right)^{3 / 2}}(0,-\sin (\omega t), \cos (\omega t))
$$

as the approximation to the local part of the Biot-Savart integral near $s=x$ for any $x \in \mathbb{R}$. The scaling factor of $\ln (\epsilon)$ makes sense, in light of the form of (15). For the region $|s-x|>\epsilon$, we keep the integral (14) as it is. We therefore approximate (14) with the integro-differential equation

$$
\psi(x)+\lambda \ln \left(\epsilon^{-1}\right) \frac{\psi^{\prime \prime}(x)}{\left(1+\psi^{\prime 2}(x)\right)^{3 / 2}}+\lambda \int_{|s-x|>\epsilon} \frac{(s-x) \psi^{\prime}(s)-(\psi(s)-\psi(x))}{\left((s-x)^{2}+(\psi(s)-\psi(x))^{2}\right)^{3 / 2}} d s=0 .
$$

Recall that the planar vortex filament obtained under the LIA is naturally bounded in terms of deviation from the reference axis. In other words, for the solution $\psi$ valid under LIA, $\psi$ is bounded. In particular, it may be shown that $\psi<\sqrt{2}[13]$ in order to have a periodic solution in space. Making a similar assumption for the non-local case, let us assume that the maximal deviation of the filament from the reference axis is $0<A<1$, so that $|\psi(x)| \leq A$ for all $x \in \mathbb{R}$.

Under the assumption of a small amplitude solution $\psi$, it makes sense to consider a perturbation solution in the parameter $A$. However, as seen in Van Gorder [13], secular terms arising from regular perturbation cause blow-up of the approximations to the LIA solution. As discussed in Van Gorder [13], an effective way to overcome such difficulties would be to introduce a strained parameter which also depends on the parameter $A$. From the form of the equation (17), we consider an expansion

$$
\psi(x)=A \Psi_{0}(\chi)+A^{3} \Psi_{1}(\chi)+O\left(A^{5}\right),
$$

where

$$
\chi=\xi\left(1+\eta A^{2}+O\left(A^{4}\right)\right) x .
$$

Without loss of generality, we take $\Psi_{0}(0)=1, \Psi_{0}^{\prime}(0)=0$ so that the maximal amplitude of $\psi$ occurs at $x=0$. We also take $\Psi_{1}(0)=\Psi_{1}^{\prime}(0)=0$. The parameter $\eta$ will be selected in order to remove secular terms. Placing (18) into integro-differential equation (17), we find that

$$
\xi^{2} \lambda \ln \left(\epsilon^{-1}\right) \frac{d^{2} \Psi_{0}}{d \chi^{2}}+\Psi_{0}+\lambda I_{0}\left[\Psi_{0}\right]=0
$$




$$
\xi^{2} \lambda \ln \left(\epsilon^{-1}\right) \frac{d^{2} \Psi_{1}}{d \chi^{2}}+\Psi_{1}+\lambda I_{0}\left[\Psi_{1}\right]=\xi^{2} \lambda \ln \left(\epsilon^{-1}\right)\left\{\frac{3}{2} \xi^{2}\left(\frac{d \Psi_{0}}{d \chi}\right)^{2}-2 \eta\right\} \frac{d^{2} \Psi_{0}}{d \chi^{2}}-\lambda I_{1}\left[\Psi_{0}\right],
$$

where the integral operators $I_{0}$ and $I_{1}$ are given by

$$
\begin{gathered}
I_{0}[u(\chi)]=\int_{\epsilon}^{\infty} \frac{u^{\prime}(\chi+\sigma)-u^{\prime}(\chi-\sigma)}{\sigma^{2}} d \sigma-\int_{\epsilon}^{\infty} \frac{u(\chi+\sigma)+u(\chi-\sigma)-2 u(\chi)}{\sigma^{3}} d \sigma, \\
I_{1}[u(\chi)]= \\
\frac{3}{2} \int_{\epsilon}^{\infty} \frac{\{u(\chi-\sigma)-u(\chi)\} u^{\prime}(\chi-\sigma)}{\sigma^{4}} d \sigma-\frac{3}{2} \int_{\epsilon}^{\infty} \frac{\{u(\chi+\sigma)-u(\chi)\} u^{\prime}(\chi+\sigma)}{\sigma^{4}} d \sigma \\
+\frac{3}{2} \int_{\epsilon}^{\infty} \frac{\{u(\chi-\sigma)-u(\chi)\}^{2}}{\sigma^{5}} d \sigma+\frac{3}{2} \int_{\epsilon}^{\infty} \frac{\{u(\chi+\sigma)-u(\chi)\}^{2}}{\sigma^{5}} d \sigma .
\end{gathered}
$$

We shall pick $\xi$ from (20) and then $\eta$ to remove secular terms from (21). We find

$$
\xi=\frac{1}{\sqrt{\lambda \ln \left(\epsilon^{-1}\right)}} \sqrt{1-\lambda \frac{1+\epsilon^{2} \operatorname{Ci}(\epsilon)-\epsilon \sin (\epsilon)-\cos (\epsilon)}{\epsilon^{2}}},
$$

where we have the asymptotic scaling

$$
1-\lambda \frac{1+\epsilon^{2} \operatorname{Ci}(\epsilon)-\epsilon \sin (\epsilon)-\cos (\epsilon)}{\epsilon^{2}}=1-\lambda\left(\ln (\epsilon)+\gamma-\frac{1}{2}-\frac{1}{8} \epsilon^{2}+O\left(\epsilon^{4}\right)\right)
$$

for small $\epsilon>0$. This gives the order zero term $\Psi_{0}(\chi)=\cos (\chi)$. Meanwhile, to remove the secular terms from $(21)$ which result from the choice of $\Psi_{0}(\chi)$, we must pick

$$
\eta=\frac{3}{16} \xi^{2}=\frac{3}{16 \lambda \ln \left(\epsilon^{-1}\right)}\left(1-\lambda \frac{1+\epsilon^{2} \operatorname{Ci}(\epsilon)-\epsilon \sin (\epsilon)-\cos (\epsilon)}{\epsilon^{2}}\right) .
$$

With secular terms removed, the solution to (21) takes the form

$$
\Psi_{1}(\chi)=a(\cos (\chi)-\cos (2 \chi))+b(\cos (\chi)-\cos (3 \chi))
$$

and we find that

$$
\begin{gathered}
a=\frac{\lambda\left\{\epsilon^{4}[\mathrm{Ci}(\epsilon)-8 \mathrm{Ci}(2 \epsilon)]+\left(6+\epsilon^{2}\right) \cos (\epsilon)+\left(2 \epsilon-\epsilon^{3}\right) \sin (\epsilon)-\left(3+2 \epsilon^{2}\right) \cos (2 \epsilon)+\left(4 \epsilon^{3}-2 \epsilon\right) \sin (2 \epsilon)-3\right\}}{8 \epsilon^{2}\left\{3 \epsilon^{2}+\lambda\left(4 \epsilon^{2}[\operatorname{Ci}(\epsilon)-\operatorname{Ci}(2 \epsilon)]+2 \epsilon[\sin (2 \epsilon)-2 \sin (\epsilon)]+\cos (2 \epsilon)-4 \cos (\epsilon)+3\right)\right\}}, \\
b=\frac{3}{8 \lambda \epsilon^{2} \ln \left(\epsilon^{-1}\right)} \frac{\left(\epsilon^{2}+\lambda+\epsilon^{2} \operatorname{Ci}(\epsilon) \lambda-\epsilon \sin (\epsilon) \lambda-\cos (\epsilon) \lambda\right)^{2}}{8 \epsilon^{2}+\lambda\left(8+9 \epsilon^{2}[\mathrm{Ci}(\epsilon)-\operatorname{Ci}(3 \epsilon)]+3 \epsilon[\sin (3 \epsilon)-3 \sin (\epsilon)]+\cos (3 \epsilon)-9 \cos (\epsilon)\right)} .
\end{gathered}
$$

For small $\epsilon>0$, these coefficients behave like

$$
\begin{aligned}
a & =\lambda \frac{84 \gamma+84 \ln (\epsilon)+96 \ln (2)-133}{384 \ln (2) \lambda-288}+O\left(\epsilon^{2}\right), \\
b & =\frac{3}{32 \lambda \ln (\epsilon)} \frac{[2+\lambda(2 \gamma-1+2 \ln (\epsilon))]^{2}}{9 \ln (3) \lambda-8}+O\left(\epsilon^{2}\right) .
\end{aligned}
$$


We therefore have the perturbation solution

$$
\psi(x)=A \cos (\mu x)+a A^{3}(\cos (\mu x)-\cos (2 \mu x))+b A^{3}(\cos (\mu x)-\cos (3 \mu x))+O\left(A^{5}\right),
$$

where

$$
\mu=\xi+\frac{3}{16} \xi^{3} A^{2}+O\left(A^{4}\right)
$$

The space period of a non-local planar filament under (17) is then approximated for small $A>0$ by

$$
\tau=\frac{2 \pi}{\mu}=\frac{2 \pi}{\xi+\frac{3}{16} \xi^{3} A^{2}}+O\left(A^{4}\right) .
$$

While the planar filament arising from the LIA (with all non-local influences suppressed) can be given in terms of elliptic functions in an exact implicit form, we shall consider the Poincaré Lindstedt method again, since this permits one to directly compare the LIA solution to the solution obtained under the non-local formulation, above. The Poincaré - Lindstedt method used here was applied to the standard LIA in the case of a planar vortex filament in Van Gorder [13]. In that paper, the $\epsilon$ influence was suppressed in a composite parameter, so we redo the calculations here in order to preserve the parameters $\lambda$ and $\epsilon$. If we solve (20)-(21) by taking $I_{0} \equiv I_{1} \equiv 0$, then we obtain the LIA results. We find

$$
\begin{gathered}
\xi_{\text {LIA }}=\frac{1}{\sqrt{\lambda \ln \left(\epsilon^{-1}\right)}}, \\
\eta_{\text {LIA }}=\frac{3}{16} \xi_{\text {LIA }}^{2}=\frac{3}{16 \lambda \ln \left(\epsilon^{-1}\right)} .
\end{gathered}
$$

With this, we have

$$
\psi_{\mathrm{LIA}}(x)=A \cos \left(\mu_{\mathrm{LIA}} x\right)+\frac{3}{64 \lambda \ln \left(\epsilon^{-1}\right)} A^{3}\left\{\cos \left(\mu_{\mathrm{LIA}} x\right)-\cos \left(3 \mu_{\mathrm{LIA}} x\right)\right\}+O\left(A^{5}\right),
$$

where

$$
\mu_{\mathrm{LIA}}=\frac{1}{\sqrt{\lambda \ln \left(\epsilon^{-1}\right)}}\left\{1+\frac{3}{16 \lambda \ln \left(\epsilon^{-1}\right)} A^{2}\right\}+O\left(A^{4}\right) .
$$

The space period of a planar filament under the LIA in our scaled coordinates is then approximated for small $A>0$ by

$$
\tau_{\mathrm{LIA}}=\frac{2 \pi}{\mu_{\mathrm{LIA}}}=\frac{2 \pi}{\xi_{\mathrm{LIA}}+\frac{3}{16} \xi_{\mathrm{LIA}}^{3} A^{2}}+O\left(A^{4}\right) .
$$

For small $\epsilon=10^{-n}, n$ a positive integer, we have that

$$
\xi=\frac{1}{\sqrt{\lambda \ln \left(\epsilon^{-1}\right)}} \sqrt{1+[n \ln (10)+0.5-\gamma] \lambda+O\left(10^{-2 n}\right)}>\frac{1}{\sqrt{\lambda \ln \left(\epsilon^{-1}\right)}}=\xi_{\mathrm{LIA}},
$$

and hence $\mu>\mu_{\text {LIA }}$. Therefore, in the perturbative regime, $\tau<\tau_{\text {LIA }}$. On the other hand, if $\epsilon$ is particularly large, the reverse inequalities can hold.

With this, we have shown that in the small amplitude limit, a planar vortex filament does indeed exist under the non-local Biot-Savart dynamics, and, furthermore, that such a solution is in qualitative agreement with the planar filament solution found for the local induction approximation (LIA). 


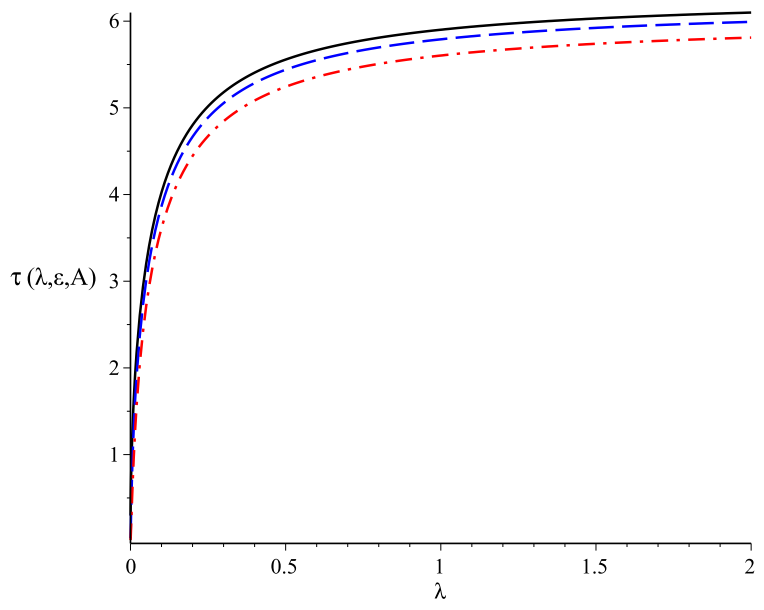

$\mathrm{A}=0.01, \varepsilon=10^{-3}--\mathrm{A}=0.3, \varepsilon=10^{-3}-\cdot-\mathrm{A}=0.5, \varepsilon=10^{-3}$

(a)

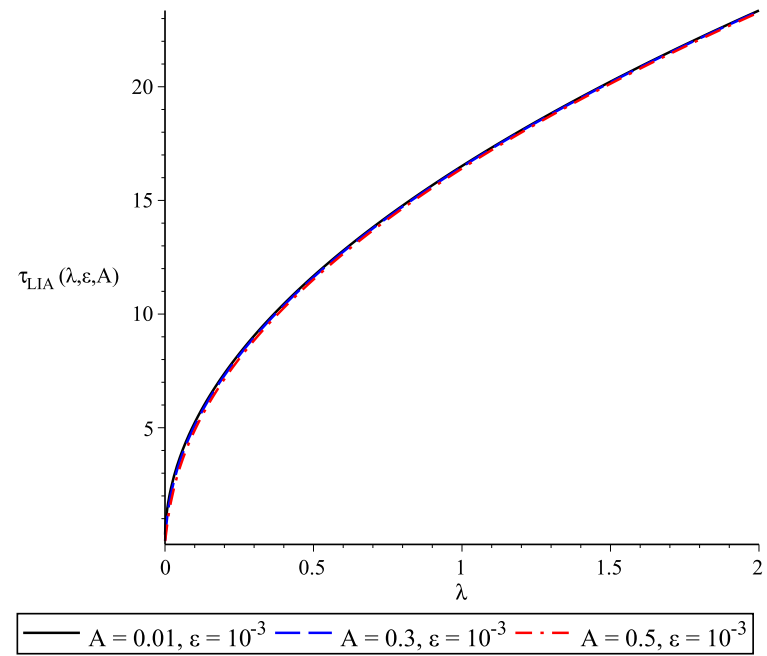

(b)

Figure 1: Plot of the spatial period of planar vortex filaments (a) with Biot-Savart terms, (b) under the LIA. We fix $\epsilon=10^{-3}$ and, for various choices of $A$, we plot the spatial period over the composite parameter $\lambda=\frac{\Gamma}{4 \pi \mid \omega}$, where $\omega$ is the rotational velocity of the filament and $\Gamma$ measures the strength of the circulation. Both solutions increase with $\lambda$, although the LIA solutions increase much more quickly with $\lambda$. Observe that an increase in the amplitude, $A$, will result in a decrease in the spatial period for both the Biot-Savart and the LIA planar filaments.

In Figures 1 and 2, we demonstrate certain qualitative features of the planar filament solutions. Since the difference in any two solutions will essentially lie in their spatial periods, we provide plots of $\tau$ as a function of the composite parameter $\lambda=\frac{\Gamma}{4 \pi|\omega|}$ (recall that $\omega$ is the rotational velocity, while $\Gamma$ is the strength of the circulation), the amplitude of the maximal displacements from the reference axis, $A$, and the length scale of the regularization, $\epsilon$.

If $|\omega|>>\Gamma$, then $\lambda<<1$ and we have that the spatial period is very small. Therefore, rapidly rotating planar vortex filaments should have relatively small spatial periods. On the other hand, if $|\omega|<<\Gamma$, then $\lambda>>1$, and the spatial period becomes larger. So, in the case where the strength of the circulation is much greater than the rate of rotational motion of the filament, we expect the spatial period to be larger. For both the regularized Biot-Savart solution and the LIA solution, the qualitative behavior of solutions is the same, yet for larger $\lambda$, the spatial period of the LIA solutions becomes much larger than that of a corresponding Biot-Savart solution. The non-local terms therefore appear to smooth the solutions in a sense, with the growth of the spatial period bounded in $\lambda$. Therefore, a sharp increase in the intensity of the circulation does not drastically increase the period of the Biot-Savart solutions by very much. Observe that an increase in the amplitude, $A$, will result in a decrease in the spatial period for both the Biot-Savart and the LIA planar filaments. We find that an increase in the magnitude of the regularization length scale $\epsilon$ results in a decrease in the period of both solutions. Note that all of these findings are valid for sufficiently small amplitude displacements, $A$, due to the perturbation approach used.

When $\lambda \rightarrow 0$, i.e. when $|\omega|>>\Gamma$, both spatial periods become arbitrarily small. Furthermore, Figures 1 and 2 suggest that the spatial period of the Biot-Savart solutions seemingly remains 


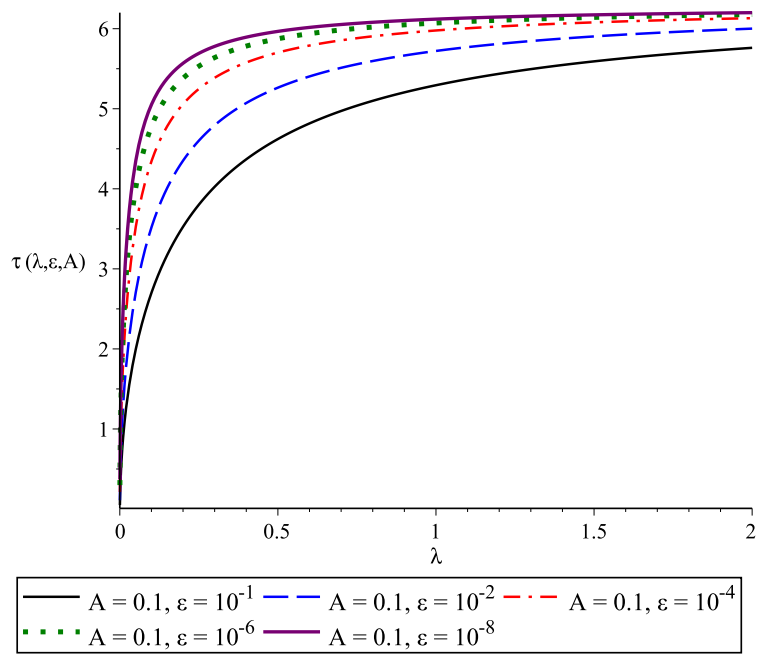

(a)

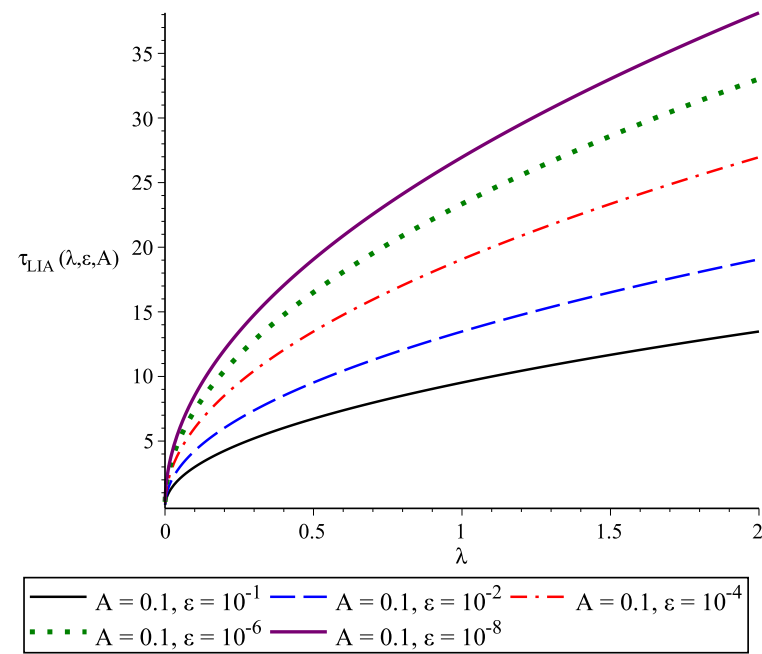

(b)

Figure 2: Plot of the spatial period of planar vortex filaments (a) with Biot-Savart terms, (b) under the LIA. We fix $A=0.1$ and, for various choices of regularization length scale $\epsilon$, we plot the spatial period over the composite parameter $\lambda=\frac{\Gamma}{4 \pi|\omega|}$. We find that an increase in the magnitude of the regularization length scale $\epsilon$ results in a decrease in the period of both solutions. Again, the LIA solutions exhibit much larger spatial periods than do the Biot-Savart solutions when $\lambda$ is sufficiently large.

bounded as $\lambda \rightarrow \infty$ (i.e., when $|\omega|<<\Gamma$ ). To demonstrate this, we can obtain the large- $\lambda$ asymptotics for $\xi$. We see from (24) that

$\xi=\sqrt{\frac{1+\epsilon^{2} \operatorname{Ci}(\epsilon)-\epsilon \sin (\epsilon)-\cos (\epsilon)}{\epsilon^{2} \ln (\epsilon)}+\frac{1}{\ln \left(\epsilon^{-1}\right)} \lambda^{-1}}=\sqrt{\frac{1+\epsilon^{2} \operatorname{Ci}(\epsilon)-\epsilon \sin (\epsilon)-\cos (\epsilon)}{\epsilon^{2} \ln (\epsilon)}}+O\left(\lambda^{-1 / 2}\right)$,

hence

$$
\xi \rightarrow \xi_{0}(\epsilon)=\sqrt{\frac{1+\epsilon^{2} \mathrm{Ci}(\epsilon)-\epsilon \sin (\epsilon)-\cos (\epsilon)}{\epsilon^{2} \ln (\epsilon)}}
$$

as $\lambda \rightarrow \infty$. Therefore, $\xi$ is finite in the limit $\lambda \rightarrow \infty$. Furthermore, for small $\epsilon$, we can approximate this limit $\xi_{0}(\epsilon)$ like

$$
\xi_{0}(\epsilon)=\sqrt{1+\frac{\gamma-\frac{1}{2}}{\ln (\epsilon)}}+O(\epsilon) .
$$

Therefore, the spatial period of a regularized Biot-Savart solution must behave like

$$
\tau=2 \pi\left\{\sqrt{1+\frac{\gamma-\frac{1}{2}}{\ln (\epsilon)}}+\frac{3}{16} A^{2}\right\}^{-1}+O\left(\left(\frac{\gamma-\frac{1}{2}}{\ln (\epsilon)}\right)^{3 / 2}\right)+O\left(\lambda^{-1 / 2}\right)+O\left(A^{4}\right)
$$

for very large $\lambda$, very small $\epsilon$, and small $A$. This result is in agreement with what we see in Figures 1 and 2 for the regularized Biot-Savart solutions, as for larger $\lambda$ the spatial period takes an asymptotic value below $2 \pi$, with this value decreasing as $A$ increases. 
On the other hand, we have that $\xi_{\text {LIA }}=O\left(\lambda^{-1 / 2}\right)$, and we therefore have

$$
\tau_{\text {LIA }}=2 \pi \sqrt{\lambda \ln \left(\epsilon^{-1}\right)}\left\{1+\frac{3 A^{2}}{16 \lambda \ln \left(\epsilon^{-1}\right)}\right\}^{-1}+O\left(A^{4}\right)=2 \pi \sqrt{\lambda \ln \left(\epsilon^{-1}\right)}+O\left(A^{2} \lambda^{-1}\right) .
$$

Hence, for fixed $\epsilon>0$ and $A>0, \tau_{\text {LIA }}=O\left(\lambda^{1 / 2}\right)$ for large $\lambda$, which is why the spatial period of the LIA solutions is asymptotically increasing with $\lambda$. Physically, of course, there will be a bound on the value of $\lambda$, so the spatial period of the LIA solutions will be bounded. However, these results explain why the spatial period of the LIA solutions is much larger than that of the regularized Biot-Savart solutions for large $\lambda$.

Therefore, when the rotational velocity is much stronger than the circulation of the vortex filament, we expect the Biot-Savart and LIA solutions to show strong qualitative agreement. On the other hand, if the rotational velocity is rather weak compared with the strength of the circulation, then the LIA can drastically overestimate the spatial period of the planar vortex filament solutions. A change in either the amplitude or the regularization length scale will have a similar qualitative change on either the regularized Biot-Savart or the LIA planar filaments.

\section{Conclusions}

A planar solution has been constructed for the full non-local Biot-Savart dynamics governing the self-induced motion of a vortex filament. Under the assumption of a planar vortex filament solution with arbitrary (unknown) spatial structure determined by a function $\psi(x)$, the Biot-Savart dynamics reduce to a stationary (independent of time) integral equation for the unknown function $\psi(x)$. (Actually, since the formulation also depends on a $\psi^{\prime}$ under the integral, this equation is a type of integro-differential equation.) The integral in this formulation is then approximated near a singularity of logarithmic order by the LIA, whereas away from the singularity the integral form is maintained. Therefore, there is a part of the solution due to the LIA, and another non-local part. This solution is interesting in a mathematical sense, due to the fact that it provides us with an accurate approximation to a solution of the singular and nonlinear integro-differential equation (14). Even the numerical solution of such an integro-differential equation would be challenging. The results suggest that at the very least some of the interesting stationary solutions present under the LIA can also be extended to the Biot-Savart formulation. Generalized rotating filament solutions, which generalize the planar filaments considered here, are also possible and would be an interesting area for future work. Under the LIA, such generalized purely rotating vortex filament solutions were recently studied in Van Gorder [22]. A wide variety of solutions were described, and it would be interesting to see which of these solutions carry over to the non-local Biot-Savart model.

Physically, the approximate analytical solution obtained through an application of the Poincaré - Lindstedt method is quite useful. While we assume that the deviations $A$ from the central axis are sufficiently small, this is physically reasonable in light of the fact that the planar filaments only exist for the LIA when $A<\sqrt{2}$. So, the large $A$ case is not physically interesting. For the solutions obtained in Van Gorder [13], the perturbation solutions were useful for $A<0.7$. The approximate solutions suggest that the spatial structure of the planar filaments found here for the Biot-Savart dynamics is relatively unchanged from the structure of the solutions under LIA, at least in the small amplitude regime.

For a helical filament, the Biot-Savart formulation and the LIA result in similar forms of the translational and rotational velocity as functions of the wave number and amplitude of the helical 
filament. Therefore, the structure of such solutions is preserved in a qualitative sense under the LIA. Similarly, we have shown in this paper that the structure of a planar filament is preserved between the Biot-Savart formulation and the LIA. In the planar case, one always obtains a single equation governing an unknown function (in contrast to the helical case, where both formulations give equations for the parameters measuring translational and rotational velocities); the difference being that the non-local formulation gives an integral equation, while the local formulation gives a differential equation. It is then interesting to ask whether this type of functional dependence is always preserved under the approximation of Biot-Savart dynamics with the LIA, at least for those cases where vortex filament solutions can be expressed analytically.

The quantum analogue to the LIA was provided by Schwarz [10]. It was shown in Van Gorder [23] that an exact planar solution does not exist under the quantum LIA (unless there are extraordinary special experimental conditions) due to torsion effects. (The planar filament is a torsionless solution.) However, it is possible to study filaments which initially are planar and gradually evolve due to the quantum LIA. Such solutions essentially involve two unknown functions (as opposed to the one unknown function which is to be determined in the planar case). Translational effects for such solutions are no longer zero, but they can be shown to be small (of the order $\alpha^{2}$, where $\alpha$ is the larger of the two small mutual friction parameters). Therefore, between [23] and the present paper, it is reasonable to expect that analogous solutions (which are planar at $t=0$ and gradually evolve into non-planar structures) can be found in the non-local form of the quantum model, where LIA terms are replaced by Biot-Savart integrals.

\section{References}

[1] Arms, R.J. \& Hama, F.R. 1965 Localized-induction concept on a curved vortex and motion of an elliptic vortex ring. Phys. Fluids 8, 553.

[2] Da Rios, L.S. 1906 Sul moto d'un liquido indefinite con un filetto vorticoso di forma qualunque. Rend. Circ. Mat. Palermo 22, 117.

[3] Hasimoto, H. 1972 A soliton on a vortex filament. J. Fluid Mech. 51, 477.

[4] Kida, S. 1981 A vortex filament moving without change of form. J. Fluid Mech. 112, 397.

[5] Ricca, R. L., Samuels, D. C. , \& Barenghi, C. F. 1999 Evolution of vortex knots. Journal of Fluid Mechanics 391, 29.

[6] Sonin, E. B. 2012 Dynamics of helical vortices and helical-vortex rings. EPL (Europhysics Letters) 97, 46002.

[7] Ricca, R. L. 1996 The contributions of Da Rios and Levi-Civita to asymptotic potential theory and vortex filament dynamics. Fluid Dynamics Research 18, 245.

[8] Hasimoto, H. 1971 Motion of a Vortex Filament and its relation to Elastica. Journal of the Physical Society of Japan 31, 293.

[9] Gutiérrez, S., Rivas, J., Vega, L. 2003 Formation of Singularities and Self-Similar Vortex Motion Under the Localized Induction Approximation. Communications in Partial Differential Equations 28, 927. 
[10] Schwarz, K. W. 1985 Three-dimensional vortex dynamics in superfluid ${ }^{4}$ He: Line-line and line-boundary interactions. Phys. Rev. B 31, 5782.

[11] Svistunov, B. V. 1995 Superfluid turbulence in the low-temperature limit. Phys. Rev. B 52, 3647 .

[12] Van Gorder, R.A. 2012 Integrable stationary solution for the fully nonlinear local induction equation describing the motion of a vortex filament, Theor. Comput. Fluid Dyn. 26, 591.

[13] Van Gorder, R.A. 2013 Scaling laws and accurate small-amplitude stationary solution for the motion of a planar vortex filament in the Cartesian form of the local induction approximation, Phys. Rev. E 87, 043203.

[14] Kida, S. 1982 Stability of a steady vortex filament, Journal of the Physical Society of Japan $\mathbf{5 1}, 1655$.

[15] Van Gorder, R. A. 2013 Orbital Stability for Rotating Planar Vortex Filaments in the Cartesian and Arclength Forms of the Local Induction Approximation. Journal of the Physical Society of Japan 82, 094005.

[16] Fukumoto, Y. 1997 Stationary configurations of a vortex filament in background flows, Proceedings of the Royal Society of London A 453, 1205.

[17] Umeki, M. 2010 A locally induced homoclinic motion of a vortex filament. Theor. Comput. Fluid Dyn. 24, 383.

[18] Van Gorder, R.A. 2012 Exact solution for the self-induced motion of a vortex filament in the arclength representation of the local induction approximation, Phys. Rev. E 86, 057301.

[19] Zhou, H. 1997 On the motion of slender vortex filaments. Phys. Fluids 9, 970.

[20] Boffetta, G., Celani, A., Dezzani, D., Laurie, J., Nazarenko, S. 2009 Modeling Kelvin Wave Cascades in Superfluid Helium. J. Low Temp. Phys. 156, 193.

[21] Van Gorder, R. A. 2015 Helical vortex filament motion under the non-local Biot-Savart model. Journal of Fluid Mechanics 762, 141-155.

[22] Van Gorder, R. A. 2014 General rotating quantum vortex filaments in the low-temperature Svistunov model of the local induction approximation. Phys. Fluids 26, 065105.

[23] Van Gorder, R. A. 2014 Dynamics of a planar vortex filament under the quantum local induction approximation. Proceedings of the Royal Society A 470, 20140341. 